\title{
Cardiac hemodynamic response to the 6-minute walk test in patients with intestinal carcinoma undergoing bevacizumab treatment
}

\author{
Huiling Huang ${ }^{1,2 \#}$, Yalin Cao ${ }^{3 \#}$, Yugang Dong ${ }^{1,2}$, Jiayong $\mathrm{Li}^{1,2}$, Chen Liu ${ }^{1,2}$, Marvin Owusu-Agyema ${ }^{1,2}$, \\ Yao Tong ${ }^{1,2}$, Fengjuan $\mathrm{Yao}^{4}$, Baolin $\mathrm{Chen}^{3}$, Ling $\mathrm{Li}^{3}$, Fawang $\mathrm{Du}^{3}$, Xingwei $\mathrm{Hu}^{3}$, Xing Wang ${ }^{1,2}$, \\ Yanhong Deng ${ }^{5,6}$
}

${ }^{1}$ Department of Cardiology, the First Affiliated Hospital of Sun Yat-sen University, Guangzhou, China; ${ }^{2}$ Key Laboratory on Assisted Circulation, Ministry of Health, Guangzhou, China; ${ }^{3}$ Department of Cardiology, Guizhou Provincial People’s Hospital, Guiyang, China; ${ }^{4}$ Department of Cardiac ultrasound, the First Affiliated Hospital, Sun Yat-sen University, Guangzhou, China; ${ }^{5}$ Department of Medical Oncology, the Sixth Affiliated Hospital of Sun Yat-sen University, Guangzhou, China; ${ }^{6}$ Guangdong Provincial Key Laboratory of Colorectal and Pelvic Floor Diseases, Guangzhou, China Contributions: (I) Conception and design: H Huang, Y Cao; (II) Administrative support: Y Deng, X Wang; (III) Provision of study materials or patients: Y Deng, X Wang; (IV) Collection and assembly of data: J Li, C Liu, B Chen, M Owusu-Agyema; (V) Data analysis and interpretation: H Huang, Y Cao, Y Tong, F Yao, L Li, F Du, X Hu, Y Dong; (VI) Manuscript writing: All authors; (VII) Final approval of manuscript: All authors.

\#These authors contributed equally to this work.

Correspondence to: Xing Wang, MD. Department of Cardiology, the First Affiliated Hospital of Sun Yat-sen University, 58 Zhongshan Road 2, Guangzhou, China. Email: 2368829@qq.com; Yanhong Deng, MD, PhD. Department of Medical Oncology, the Sixth Affiliated Hospital of Sun Yatsen University, 26 Erheng Road, Tianhe District, Guangzhou, China. Email: dengyanh@mail.sysu.edu.cn.

Background Exercise capacity is evaluated using the 6-minute walk test (6MWT) in various cardiovascular diseases. Bevacizumab (BEV) has been associated with significant risk of cardiovascular complications. The aim of this study was to investigate BEV-related influences on cardiac hemodynamic response to 6MWT.

Methods: We prospectively studied 24 patients with intestinal carcinoma to assess the hemodynamic response during $6 \mathrm{MWT}$, of whom eight underwent BEV treatment. Obtained data was analyzed to identify hemodynamic differences between BEV and non-BEV treated patients.

Results: Twenty-four patients with stage IV intestinal carcinoma consented to assessment after the completion of three cycles of BEV-combined chemotherapy (age, $46.4 \pm 16.7$ years) or standard chemotherapy alone (age, 56.4 \pm 13.7 years). In comparison with non-BEV treated patients, BEV-treated patients walked less (484.3 \pm 42.4 vs. 503.0 $\pm 48.2, \mathrm{P}=0.339)$. These two groups manifested similar hemodynamic response during the 6MWT. The change of hemodynamic parameters at 1 minute after completion of $6 \mathrm{MWT}$ was defined as hemodynamic parameter recovery. BEV-treated patients had significantly lower change of left cardiac work index (LCWi), cardiac index (CI), cardiac output (CO) and stroke volume (SV) after 6MWT. Interestingly, in BEV-treated patients $\mathrm{CI}$ change after $6 \mathrm{MWT}$ was predominantly related to the decrease in SV instead of heart rate (HR) as suggested by a higher standardized beta coefficient (0.883 vs. 0.657) and semi-partial correlations (0.821 vs. 0.677).

Conclusions: Estimation of hemodynamic response to 6MWT is feasible, and may provide useful information of myocardial damage in BEV-treated patients.

Keywords: Hemodynamic response; 6-minute walk test (6MWT); bevacizumab (BEV)

Submitted Feb 16, 2020. Accepted for publication Sep 10, 2020.

doi: 10.21037/apm-20-435

View this article at: http://dx.doi.org/10.21037/apm-20-435 


\section{Introduction}

Cancer incidence and mortality have been increasing in China, making cancer the leading cause of death and a major public health problem in the country (1). Colorectal cancer (CRC) accounts for over 600,000 annual deaths worldwide and more than 1.23 million new cases are reported every year (2). In 2011, the incidence and mortality of CRC in China were 23.03/100,000 and 11.11/100,000 respectively. In the past few decades, substantial progress has been made in the treatment of patients with advanced CRC (3-5). Angiogenesis is a critical regulator in tumor progression and metastasis, and anti-angiogenic drugs have been the focal topic in the field of cancer research $(6,7)$. The process is mainly driven by vascular endothelial growth factor (VEGF), high levels of circulatory VEGF predict a poor prognosis in cancer patients (8).

Bevacizumab (BEV), a humanized monoclonal antibody that binds to the VEGF, was the first biological agent of this class that has shown efficacy for the treatment of metastatic CRC (9). The combination of standard chemotherapy with BEV regimens in advanced CRC has been shown significantly increase overall survival, progression-free survival, and/or overall response rate (10). However, despite the prolonged survival rates, $\mathrm{BEV}$-associated cardiovascular toxicities have been increasingly recognized. Cardiovascular toxicity following the treatment of $\mathrm{BEV}$ might manifest as hypertension, ischemic heart disease, thromboembolic events, or congestive heart failure (11). Because of the life-threatening impact of severe cardiovascular adverse events, recognition and management of $\mathrm{BEV}$-related cardiovascular toxicity has become tightly integrated with routine cancer care. Since 2007, many patients treated with BEV have undergone prospective cardiac monitoring using regular monitoring protocol. There is an urgent need to identify affordable noninvasive methodologies that can provide early and accurate information on BEV-related cardiovascular toxicity.

Heart failure is the terminal stage of all cardiovascular diseases. Its etiology encompasses various conditions, including hypertension and ischemic heart disease. The complex interplay between hypertension, ischemic heart disease and heart failure has been a matter of interest. In the last decade several insights into the pathophysiology have led to a better understanding of mechanisms: activation of the renin-angiotensin-aldosterone system, abnormal activity of the sympathetic nervous system and overproduction of inflammatory cytokines. These neuroendocrine imbalances contribute to vascular and endothelial dysfunction, impaired cardiac output (CO) and changes in systemic vascular resistance and arterial compliance. In this regard, the measurements of hemodynamic parameters may be a valuable tool for early detection of asymptomatic cardiovascular disease.

The components of a typical clinical assessment of an asymptomatic patient include medical history, physical examination, electrocardiograph, and echocardiography. But the changes of hemodynamic parameters in response to physiological tests could not be evaluated through echocardiography. Impedance cardiography (ICG) is a reliable, well-tolerated, and non-invasive method used to dynamically obtain hemodynamic measurements, which is based on the Ohm's law. ICG has been demonstrated the further potential for cardiovascular disease detection in asymptomatic patients with cardiovascular risk factors, allowing cardiac remodeling prevention $(12,13)$. In the present study, we determined to test whether ICG during 6-minute walking test (6MWT) reveals impaired hemodynamic responses in patients with intestinal carcinoma undergoing BEV treatment. We present the following article in accordance with the reporting checklist (available at http://dx.doi.org/10.21037/apm-20-435).

\section{Methods}

\section{Study population}

We conducted an observational study in patients with intestinal carcinoma undergoing BEV treatment. Our study enrolled patients who were newly diagnosed of metastatic disease (American Joint Committee on Cancer Stage IV) by two oncologists from June 2018 to September 2019. All included patients received the standard chemotherapy of 5-FU, calcium folinate and oxaliplatin. Patients eligible for inclusion were of either sex, aged 18 years or older. Patients must have normal cardiac ejection fraction (EF), normal organs and marrow function. We excluded patients either who had prior cancer history other than CRC to avoid bias due to effects of any prior chemotherapy for other malignancies; or had any cardiovascular diseases to minimize the hemodynamic influence. The study was conducted in accordance with the Declaration of Helsinki (as revised in 2013). The study was approved by the Medical Ethics Commission of sixth Affiliated Hospital of Sun Yatsen University (No. E2014022) and informed consent was taken from all the patients.

Data on lifestyle, profession, medical and family history were obtained using structured questionnaire. On the day of examination, anthropometric measurements were 
performed in duplicate using standardized procedures. Height was measured to the nearest $0.1 \mathrm{~cm}$, body mass to the nearest $0.1 \mathrm{~kg}$, body mass index (BMI) was subsequently calculated $\left(\mathrm{kg} / \mathrm{m}^{2}\right)$. Recent biochemical profiles (last 1 month) were retracted from patients' medical records. Blood pressure was obtained according to the current recommendations using an oscillometric semiautomatic sphygmomanometer and reported as the average of two readings taken with 2 minutes interval (14). Measurements started after the patients had comfortably rested for 5 minutes in the sitting position. The size of the bladder was adjusted to the arm circumference, using a larger bladder (arm circumference $31-40 \mathrm{~cm}$ ) in obese patients.

\section{ICG and hemodynamic measurements}

The $6 \mathrm{MWT}$ was performed according the Guidelines by the American Thoracic Society (15). All subjects were tested by the same trained technician. Patients remained seated for approximately 10 minutes before the test (during consenting, electrode placement, and connection of the device).

The hemodynamic parameters were measured by using a portable, new generation, signal morphology-based impedance cardiograph with real-time wireless monitoring via a bluetooth USB adapter (PhysioFlow Enduro, Paris, France). PhysioFlow Enduro measured the changes in impedance by injecting a high frequency alternating electrical current $(66 \mathrm{kHz})$ of low magnitude $(3.8 \mathrm{~mA}$ rms.) towards the thorax between a pair of electrodes positioned on the neck and another pair positioned on xiphoïd process. By detecting and measuring the difference of thoracic impedance over time, PhysioFlow Enduro could record the systolic volume, $\mathrm{CO}$ and several other hemodynamic parameters in a noninvasive way. We prepped the skin and fresh electrodes (pre-gelled Skintact FS-50) were placed in the neck (the left base of the neck above the supraclavicular fossa, $n=2$ ) and thoracic cavity (left paraspinal muscles at the level of the xiphoid process, $n=2$; right upper chest, $\mathrm{n}=1$; and left lower chest, $\mathrm{n}=1$ ) to detect the variations of signal. The data obtained (ICG signals) were processed and hemodynamic parameters were calculated.

\section{Statistical analysis}

Continuous variables were summarized using mean and standard deviation if they follow normal distribution; otherwise, they were presented as medians and interquartile ranges. Categorical variables were presented as frequency (percentage). Differences in the baseline characteristics were compared using Student's $t$-test for normally distributed variables and the chi-square test for categorical variables. The Mann-Whitney $U$ test was used to compare continuous variables in states of non-normality. We utilized logistic regression to compare the hemodynamic response during 6MWT according to BEV use in our study population. We calculated semipartial correlations using linear regression with cardiac index (CI) change as dependent variable and heart rate (HR) change or stroke volume (SV) change as covariates. Person correlation to identify the strength of association between variables. All the $\mathrm{P}$ values were reported as two tailed. A $\mathrm{P}$ value of $<0.05$ was prespecified as indicative of statistical significance. Statistical analysis was performed using the IBM SPSS22 (IBM, NY, USA) program.

\section{Results}

During the study period, a total of 24 patients were diagnosed as metastatic CRC, of which eight patients received BEV treatment. They all received three cycles chemotherapy of 5-FU, calcium folinate and oxaliplatin. The baseline clinical stage at enrollment was IVA in 14 (58.3\%) and IVB in 10 (41.7\%). Patients' demographic and clinical data were presented in Table 1 . The type of intestinal carcinoma $(n=24)$ included colorectal carcinoma $(\mathrm{n}=9,37.5 \%)$, sigmoid colon carcinoma $(\mathrm{n}=8,33.4 \%)$ and others. The mean age was 53.1 years, and $54.2 \%$ were men. Age was $46.4 \pm 16.7$ and $56.4 \pm 13.7$ for patients treated with $\mathrm{BEV}$ and without $\mathrm{BEV}$, respectively. The average BMI was $20.8 \pm 2.4 \mathrm{~kg} / \mathrm{m}^{2}$.

Results of the traditional 6MWT measurements were shown in Table 2. Compared with subjects without BEV treatment, BEV-treated patients walked less $(484.3 \pm 42.4$ vs. 503.0 $\pm 48.2, \mathrm{P}=0.339)$. BEV-treated patients had significantly higher baseline HR $(95.1 \pm 17.4$ vs. $76.2 \pm 9.7$, $\mathrm{P}=0.02)$. HR change was obtained by subtracting the maximal HR during the walk from the value recorded at baseline. Heart rate recovery 1 (HRR1) was defined as the difference between a subject's HR at completion of $6 \mathrm{MWT}$ and at $1 \mathrm{~min}$ after completion of 6MWT. Interestingly, these two groups manifested with similar HR change (39.8 \pm 13.4 vs. $42.4 \pm 16.7, \mathrm{P}=0.717)$ and $\mathrm{HRR}$ at 1 minute (16.0 \pm 5.6 vs. $20.6 \pm 12.9, \mathrm{P}=0.346)$.

Similarly, absolute changes of hemodynamic parameters were defined as the difference between the maximal parameters during the walk and the parameters at baseline. 
Table 1 Characteristics of subjects

\begin{tabular}{lc}
\hline Characteristics & Value $(\mathrm{n}=24)$ \\
\hline Age (years) & $53.1 \pm 15.2$ \\
Sex, $\mathrm{n}(\%)$ & $13(54.2)$ \\
Male & $11(45.8)$ \\
Female & $20.8 \pm 2.4$ \\
BMI (kg/m $\left.{ }^{2}\right)$ & $6(25.0)$ \\
Smoking history, $\mathrm{n}(\%)$ & \\
Type of intestinal carcinoma, $\mathrm{n}(\%)$ & $2(8.3)$ \\
Transverse colon carcinoma & $2(8.3)$ \\
Descending colon carcinoma & $3(12.5)$ \\
Ascending colon carcinoma & $8(33.4)$ \\
Sigmoid colon carcinoma & $9(37.5)$ \\
Colorectal carcinoma & \\
Clinical stage, $\mathrm{n}(\%)$ & $14(58.3)$ \\
IVA & $10(41.7)$ \\
IVB & $3.1 \pm 0.7$ \\
LDL cholesterol (mmol/L) & $1.2 \pm 0.3$ \\
HDL cholesterol (mmol/L) & $64.8 \pm 20.0$ \\
Creatinine ( $\mu$ mol/L) & \\
\hline
\end{tabular}

BMI, body mass index; LDL, low density lipoprotein; HDL, high density lipoprotein.
Hemodynamic parameters recovery were calculated as the difference between the parameters at the sixth minute of the $6 \mathrm{MWT}$ and the parameters at the first minute of recovery. Table 3 presented the values of hemodynamic variables according to BEV-treatment and non-BEV treatment. There were no hemodynamic differences during 6MWT. When BEV-treated patients were compared with non $\mathrm{BEV}$-treated patients using binary logistic regression, SV recovery [OR (95\% CI): $0.839(0.713-0.987)]$, CO recovery [OR (95\% CI): 0.873 (0.871-0.931)], CI recovery [OR (95\% CI): 0.267 (0.072-0.983)], systemic vascular resistance index (SVRI) recovery [OR (95\% CI): 0.985 (0.970-0.990)] and left cardiac work index (LCWi) recovery [OR (95\% CI): $0.283(0.087-0.920)]$ were able to discriminate between the groups. When adjusted by age, sex and distance walked at 6 minutes, LCWi [OR (95\% CI): 0.156 (0.029-0.843)], CI [OR (95\% CI): $0.217(0.050-0.943)]$, CO [OR (95\% CI): $0.274(0.084-0.893)]$ and SV [OR (95\% CI): 0.814 (0.6630.990)] recovery at 1 minute continued to be significant predictors of the presence of BEV-treatment.

In non-BEV treated individuals, CI recovery at 1 minute was not associated with $\mathrm{HR}$ at baseline or maximal $\mathrm{HR}$ but with HR change $(\mathrm{P}=0.019)$ and $\mathrm{HRR}$ at 1 minute $(\mathrm{P}=0.08)$ (Table 4). Similarly, CI recovery at 1 minute was significantly associated with SV change and SV recovery at 1 minute. In

Table 2 Traditional parameters measured during 6MWT

\begin{tabular}{|c|c|c|c|c|}
\hline Parameters & Total & Treatment with BEV $(n=8)$ & Treatment without BEV $(n=16)$ & $P$ value \\
\hline Distance at $4 \min (\mathrm{m})$ & $343.8 \pm 42.6$ & $340.3 \pm 42.5$ & $350.6 \pm 44.9$ & 0.588 \\
\hline Distance at 6 min (m) & $490.5 \pm 44.3$ & $484.3 \pm 42.4$ & $503.0 \pm 48.2$ & 0.339 \\
\hline Distance walked at $6 \mathrm{~min}$ (\% of predicted) & $18(75.0 \%)$ & $7(87.5 \%)$ & $11(68.8 \%)$ & 0.621 \\
\hline Baseline SBP (mmHg) & $116.9 \pm 16.6$ & $114.6 \pm 16.2$ & $118.1 \pm 17.2$ & 0.637 \\
\hline $\mathrm{SBP}$ at $6 \mathrm{~min}(\mathrm{mmHg})$ & $134.8 \pm 19.7$ & $126.8 \pm 9.5$ & $138.7 \pm 22.3$ & 0.168 \\
\hline Baseline DBP (mmHg) & $70.0 \pm 10.6$ & $70.6 \pm 10.1$ & $69.8 \pm 11.2$ & 0.854 \\
\hline $\mathrm{DBP}$ at $6 \mathrm{~min}(\mathrm{mmHg})$ & $77.3 \pm 10.1$ & $80.6 \pm 13.6$ & $75.7 \pm 7.9$ & 0.270 \\
\hline HRR1 (bpm) & $19.1 \pm 11.1$ & $16.0 \pm 5.6$ & $20.6 \pm 12.9$ & 0.346 \\
\hline
\end{tabular}

*, HR change was calculated as the difference between the maximal HR during the walk and the HR at baseline. HRR1 was defined as the difference between a subject's HR at completion of 6MWT and at 1 min after completion of 6MWT. 6MWT, 6-minute walk test; BEV, bevacizumab; SBP, systolic blood pressure; DBP, diastolic blood pressure; HR, heart rate; HRR1, heart rate recovery 1. 
Table 3 Hemodynamic parameters measured during 6MWT

\begin{tabular}{|c|c|c|c|c|c|}
\hline Parameters & Total & $\begin{array}{l}\text { Treatment with BEV } \\
\qquad(\mathrm{n}=8)\end{array}$ & $\begin{array}{l}\text { Treatment without } \\
\text { BEV }(n=16)\end{array}$ & $\begin{array}{c}\text { P value } \\
\text { (unadjusted) }\end{array}$ & $\begin{array}{l}\text { P value } \\
\text { (adjusted) }\end{array}$ \\
\hline Baseline SV (mL) & $59.2 \pm 12.8$ & $52.2 \pm 5.8$ & $62.7 \pm 13.9$ & 0.053 & \\
\hline SV recovery at 1 minute $(\mathrm{mL})$ & $9.8 \pm 9.7$ & $3.1 \pm 6.3$ & $13.1 \pm 9.7$ & 0.034 & 0.046 \\
\hline Peak CO (L/min) & $9.5 \pm 2.4$ & $8.7 \pm 1.5$ & $9.8 \pm 2.7$ & 0.292 & \\
\hline $\mathrm{CO}$ recovery at 1 minute (L/min) & $2.4 \pm 1.4$ & $1.5 \pm 0.7$ & $2.8 \pm 1.5$ & 0.043 & 0.032 \\
\hline CO change (L/min) & $4.7 \pm 2.0$ & $3.8 \pm 1.4$ & $5.1 \pm 2.2$ & 0.134 & \\
\hline $\mathrm{Cl}$ change $\left(\mathrm{L} / \mathrm{min} / \mathrm{m}^{2}\right)$ & $3.0 \pm 1.3$ & $2.5 \pm 0.8$ & $3.3 \pm 1.5$ & 0.157 & \\
\hline Baseline EF (\%) & $61.4 \pm 13.0$ & $65.7 \pm 9.9$ & $59.3 \pm 14.1$ & 0.262 & \\
\hline Peak EF (\%) & $70.6 \pm 15.6$ & $68.2 \pm 17.5$ & $75.4 \pm 10.1$ & 0.298 & \\
\hline EF recovery at 1 minute (\%) & $3.3 \pm 5.8$ & $2.6 \pm 6.7$ & $3.6 \pm 5.5$ & 0.686 & \\
\hline EF change (\%) & $9.2 \pm 4.1$ & $9.7 \pm 1.9$ & $8.9 \pm 4.9$ & 0.686 & \\
\hline Baseline SVRI (dyn.s/cm55/m²) & $2,240.0 \pm 450.4$ & $2,086.8 \pm 351.3$ & $2,316.6 \pm 484.4$ & 0.247 & \\
\hline Peak SVRI (dyn·s $/ \mathrm{cm}^{5} / \mathrm{m}^{2}$ ) & $1,381.0 \pm 420.2$ & $1,298.5 \pm 208.7$ & $1,422.2 \pm 494.9$ & 0.509 & \\
\hline LCWi change $\left(\mathrm{kg} \cdot \mathrm{m} / \mathrm{m}^{2}\right)$ & $3.4 \pm 1.5$ & $2.8 \pm 1.1$ & $3.7 \pm 1.6$ & 0.168 & \\
\hline
\end{tabular}

Absolute changes of hemodynamic parameters were defined as the difference between the maximal parameters during the walk and the parameters at baseline. Hemodynamic parameters recovery were calculated as the difference between the parameters at the sixth minute of the 6MWT and the parameters at the first minute of recovery. *, $\mathrm{P}$ value was adjusted for age, sex and 6-minute walking distance. 6MWT, 6-minute walk test; BEV, bevacizumab; SV, stroke volume; CO, cardiac output; CI, cardiac index; EF, ejection fraction; SVRI, systemic vascular resistance index; LCWi, left cardiac work index.

patients treated with BEV, CI recovery was predominantly associated with the recovery in SV rather than HR as suggested by a higher standardized beta coefficient $(0.883$ vs. 0.657$)$ and semi-partial correlations (0.821 vs. 0.677$)$.

\section{Discussion}

Although the $6 \mathrm{MWT}$ is an inexpensive, reproducible and safe method for assessing the functional exercise capacity in patients with different cardiovascular diseases, it has limitations both in clinical practice and research $(16,17)$. We firstly estimated the hemodynamic response to $6 \mathrm{MWT}$ in patients with intestinal carcinoma undergoing BEV treatment. In the present study, the distance walked during the 6WMT could not discriminate these two groups. However, BEV-treated patients showed smaller decrease 
Table 4 Univariate correlations between CI recovery and other parameters

\begin{tabular}{lcc}
\hline Parameters & Treatment with BEV $(\mathrm{n}=8)$ & Treatment without BEV $(\mathrm{n}=16)$ \\
\hline Distance at 6 min $(\mathrm{m})$ & 0.033 & 0.170 \\
Baseline HR $(\mathrm{bpm})$ & -0.723 & -0.480 \\
Peak HR $(\mathrm{bpm})$ & 0.538 & 0.269 \\
HR change $(\mathrm{bpm})$ & 0.156 & $0.578^{\star}$ \\
HRR1 $(\mathrm{bpm})$ & $0.657^{*}$ & $0.634^{*}$ \\
Baseline SV $(\mathrm{mL})$ & 0.470 & 0.300 \\
Peak SV $(\mathrm{mL})$ & $0.850^{\star *}$ & 0.440 \\
SV change $(\mathrm{mL})$ & $0.877^{\star *}$ & $0.533^{*}$ \\
SV recovery at 1 minute $(\mathrm{mL})$ & $0.883^{\star *}$ & $0.668^{\star *}$ \\
\hline
\end{tabular}

*, $\mathrm{P}<0.05 ;{ }^{* *}, \mathrm{P}<0.01$. Cl, cardiac index; BEV, bevacizumab; HR, heart rate; HRR1, heart rate recovery 1 ; SV, stroke volume.

in hemodynamic parameters (SV, CO, CI, SVRI, LCWi; all $\mathrm{P}<0.05)$ during the first 1 minute of recovery. For BEVtreated patients, recovery in $\mathrm{SV}$ was of critical importance in explaining the CI recovery during the test. We showed it was possible to incorporate ICG as a part of the traditional $6 \mathrm{MWT}$, as it could provide noninvasive and real-time estimations of hemodynamic parameters during $6 \mathrm{MWT}$.

VEGF is important for the repair of endothelial cells and nitrous oxide production (18). Inhibition of VEGF could increase the risk of cardiac ischemic events, there have been postmarketing reports of adverse cardiac ischemic events associated with the use of $\mathrm{BEV}(19,20)$. CI is commonly impaired during activities in early stage of ischemic heart disease, despite relatively normal values at rest. During 6MWT, BEV-treated patients walked less distance, had lower SV and CI recovery values when compared with non $\mathrm{BEV}$-treated subjects. The attenuated rise and decline in CI might be because of myocardial ischemia and autonomic imbalance alteration that affected the SV. In support of this speculation, our study found that variations in $\mathrm{SV}$ were of critical importance in explaining the CI change during the test.

$\mathrm{SV}$ is the difference between end-diastolic volume and end-systolic volume. The SV reserve is based on the reserve in systolic phase and reserve in diastolic phase. Reserve in systolic phase is achieved by increasing myocardial contractility, while diastolic reserve is achieved by increasing end-diastolic volume. As the diastolic volume is limited, SV reserve mainly depends on the reserve in systolic phase. $\mathrm{BEV}$ decreases endothelial cell renewal capacity, exposes pro-coagulant subendothelial tissue and reduces production of nitrous oxide, which promotes myocardial ischemia. One study reported a case of 54-year-old female patient developed microvascular angina after a series of BEVcontaining chemotherapeutic regimen (21). The patient's ECG indicated the presence of myocardial ischemia, which they found no significant stenosis in the epicardial coronary arteries on her coronary angiography. In this study, SV in BEV-treated patients did not increase as much as in subjects without BEV treatment at peak exercise. We implied that certain impairment of exercise capacity could be demonstrated by insufficient SV during the 6MWT. During exercise, the balance shifts towards a withdrawal of vagal tone and an increase in sympathetic activity. Higher sympathetic activity leads to accelerated HR and increased myocardial contractile force, which subsequently increases SV. Immediately after cessation of exercise, stoke volume was reduced, which was caused by sympathetic withdrawal and vagal reactivation. Vagal activity takes to recover depends on exercise intensity, type and duration of exercise, cardiorespiratory fitness. Faster vagal recovery is associated with better physical fitness and cardiovascular health. Impaired vagal recovery correlates with poorer prognoses for several clinical conditions.

In this study, we showed hemodynamic response to $6 \mathrm{MWT}$ provided by ICG might trace with early cardiac injury, supporting that this test was worthy of further investigations to better clarify its clinical role in cancer patients.

Our study has limitations. First, our study was conducted with relatively small sample size. We did not evaluate the hemodynamic parameters before the chemotherapy. 
Third, this was an observational study without controlled intervention to evaluate the hemodynamic impact of $\mathrm{BEV}$ in patients with intestinal carcinoma. Further study with a longer follow-up design is required to elucidate the impact of BEV on long-term hemodynamic changes.

\section{Acknowledgments}

Funding: This work was supported by National Key R\&D Program (2017YFC0909301), Natural Science Foundation of China (No. 81500279, No. 81370338, No. 81470511, No. 81570354 and No. 81560056), Guangdong Natural Science Foundation (No. S2013020012578, No. 2014A030313083 and No. 2015A030313111), Science and Technology Project of Guangdong Province and Guangzhou (No. 2014A021212438 and No. 201610010125), Science and Technology Project of Guizhou Province \{No. [2017]1103\}, Science and Technology Project of Health Planning Commission of Guizhou Province (No. gzwjkj2018-1-005), Program for Training Outstanding Young Scientific and Technological Talents of Guizhou Province \{No. Qian Kehe Platform Talents[2019]5662\}.

\section{Footnote}

Reporting Checklist: The authors have completed the STROBE reporting checklist. Available at http://dx.doi. org/10.21037/apm-20-435

Data Sharing Statement: Available at http://dx.doi. org/10.21037/apm-20-435

Conflicts of Interest: All authors have completed the ICMJE uniform disclosure form (available at http://dx.doi. org/10.21037/apm-20-435). The authors have no conflicts of interest to declare.

Ethical Statement: The authors are accountable for all aspects of the work in ensuring that questions related to the accuracy or integrity of any part of the work are appropriately investigated and resolved. The study was conducted in accordance with the Declaration of Helsinki (as revised in 2013). The study was approved by the Medical Ethics Commission of sixth Affiliated Hospital of Sun Yatsen University (No. E2014022) and informed consent was taken from all the patients.

Open Access Statement: This is an Open Access article distributed in accordance with the Creative Commons Attribution-NonCommercial-NoDerivs 4.0 International License (CC BY-NC-ND 4.0), which permits the noncommercial replication and distribution of the article with the strict proviso that no changes or edits are made and the original work is properly cited (including links to both the formal publication through the relevant DOI and the license). See: https://creativecommons.org/licenses/by-nc-nd/4.0/.

\section{References}

1. Chen $\mathrm{W}$, Zheng R, Baade PD, et al. Cancer statistics in China, 2015. CA Cancer J Clin 2016;66:115-32.

2. Chen $\mathrm{W}$, Zheng $\mathrm{R}$, Zeng $\mathrm{H}$, et al. Annual report on status of cancer in China, 2011. Chin J Cancer Res 2015;27:2-12.

3. Taieb J, André T, Auclin E. Refining adjuvant therapy for non-metastatic colon cancer, new standards and perspectives. Cancer Treat Rev 2019;75:1-11.

4. Benson AB, Venook AP, Al-Hawary MM, et al. NCCN guidelines insights: colon cancer, version 2.2018. J Natl Compr Canc Netw 2018;16:359-69.

5. Cremolini C, Loupakis F, Antoniotti C, et al. FOLFOXIRI plus bevacizumab versus FOLFIRI plus bevacizumab as first-line treatment of patients with metastatic colorectal cancer: updated overall survival and molecular subgroup analyses of the open-label, phase 3 TRIBE study. Lancet Oncol 2015;16:1306-15.

6. Cavallaro U, Christofori G. Molecular mechanisms of tumor angiogenesis and tumor progression. J Neurooncol 2000;50:63-70.

7. Teleanu RI, Chircov C, Grumezescu AM, et al. Tumor angiogenesis and anti-angiogenic strategies for cancer treatment. J Clin Med 2019;9:84.

8. Poon RT, Fan ST, Wong J. Clinical implications of circulating angiogenic factors in cancer patients. J Clin Oncol 2001;19:1207-25.

9. Chen HX, Mooney M, Boron M, et al. Phase II multicenter trial of bevacizumab plus fluorouracil and leucovorin in patients with advanced refractory colorectal cancer: an NCI Treatment Referral Center Trial TRC0301. J Clin Oncol 2006;24:3354-60.

10. Ferrara N, Adamis AP. Ten years of anti-vasulcar endothelial growth factor therapy. Nat Rev Drug Discov 2016;15:385-403.

11. Totzeck M, Mincu RI, Rassaf T. Cardiovascular adverse events in patients with cancer treated with bevacizumab: a meta-analysis of more than 20000 patients. J Am Heart Assoc 2017;6:e006278. 
12. DeMarzo AP. Using impedance cardiography to detect asymptomatic cardiovascular disease in prehypertensive adults with risk factors. High Blood Press Cardiovasc Prev 2013;20:61-7.

13. Krzesiński P, Gielerak GG, Kowal JJ. A 'patient-tailored' treatment of hypertension with use of impedance cardiography: a randomized, prospective and controlled trial. Med Sci Monit 2013;19:242-50.

14. Mancia G, Fagard R, Narkiewicz K, et al. 2013 ESH/ESC guidelines for the management of arterial hypertension: the Task Force for the Management of Arterial Hypertension of the European Society of Hypertension (ESH) and of the European Society of Cardiology (ESC). Eur Heart J 2013;34:2159-219.

15. ATS Committee on Proficiency Standards for Clinical Pulmonary Function Laboratories. ATS statement: guidelines for the six-minute walk test. Am J Respir Crit Care Med 2002;166:111-7.

16. Opasich C, Pinna GD, Mazza A, et al. Six-minute walking performance in patients with moderate-to-severe heart failure; is it a useful indicator in clinical practice? Eur Heart J 2001;22:488-96.

17. Zugck C, Krüger C, Dürr S, et al. Is the 6-minute walk

Cite this article as: Huang H, Cao Y, Dong Y, Li J, Liu C, Owusu-Agyema M, Tong Y, Yao F, Chen B, Li L, Du F, Hu X, Wang X, Deng Y. Cardiac hemodynamic response to the 6 -minute walk test in patients with intestinal carcinoma undergoing bevacizumab treatment. Ann Palliat Med 2021;10(2):1362-1369. doi: 10.21037/apm-20-435 test a reliable substitute for peak oxygen uptake in patients with dilated cardiomyopathy? Eur Heart J 2000;21:540-9.

18. Ranpura V, Hapani S, Chuang J, et al. Risk of cardiac ischemia and arterial thromboembolic events with the angiogenesis inhibitor bevacizumab in cancer patients: a metaanalysis of randomized controlled trials. Acta Oncol 2010;49:287-97.

19. Giantonio BJ, Catalano PJ, Meropol NJ, et al. Bevacizumab in combination with oxaliplatin, fluorouracil, and leucovorin (FOLFOX4) for previously treated metastatic colorectal cancer: results from the Eastern Cooperative Oncology Group Study E3200. J Clin Oncol 2007;25:1539-44.

20. Price TJ, Zannino D, Wilson K, et al. Bevacizumab is equally effective and no more toxic in elderly patients with advanced colorectal cancer: a subgroup analysis from the AGITG MAX trial: an international randomised controlled trial of Capecitabine, Bevacizumab and Mitomycin C. Ann Oncol 2012;23:1531-6.

21. Katoh M, Takeda N, Arimoto T, et al. Bevacizumabrelated microvascular angina and its management with nicorandil. Int Heart J 2017;58:803-5. 